\title{
Hepatitis crónica por virus C: factores asociados a la severidad del daño histológico
}

\author{
Ivonne 0 rellana $\mathbf{N}^{1}$, Jaime Poniachik $\mathbf{T}^{1}$, G ladys Smok $\mathbf{S}^{3}$, \\ Ana María Madrid $S^{1}$, Alejandra Menéndez $A^{2}$, \\ Eduardo Tobar A $^{2}$, Javier Brahm B ${ }^{1,4}$.

\section{Factors associated with the severity of liver damage in chronic hepatitis $C$}

Background: Twenty percent of patients with chronic hepatitis $C$ evolve to cirrhosis in 10 to 20 years. The degree of steatosis and hepatic iron stores in liver biopsy increase the risk. Age, high body mass index, diabetes mellitus and alcohol consumption are factors associated to the severity of liver damage. Aim: To study the association of steatosis and increased iron stores in the liver biopsy and age, overweight, alcohol consumption and diabetes with the severity of liver damage in patients with hepatitis $C$ virus infection. Patients and methods: Retrospective study of 84 liver biopsies of patients with chronic infection with hepatitis $C$ virus were studied. The pathological appearance was classified as stage I when chronic hepatitis with mild activity without fibrosis was observed; as stage II when moderate chronic hepatitis with mild fibrosis was observed and as stage III when there was a moderate chronic hepatitis with fibrosis or cirrhosis. The amount of steatosis and iron deposition in the biopsy were also assessed. Results: Forty one percent of patients were in stage I, $32 \%$ in stage II and $27 \%$ in stage III. Patients in stage I were younger than those in stages II and III (40.7 and 52.2 years respectively, $\mathrm{p}<0,001)$. No association between the severity of liver damage and the degree of steatosis, hemosiderosis, body mass index or alcohol intake, was observed. The frecuency of diabetes mellitus increased along with pathological staging $(3,15$ and $30 \%$ in stages I, II and III, respectively, $\mathrm{p}<0,05$ ). Conclusions: This study confirms that severity of chronic hepatitis $\mathrm{C}$ is associated with age and the presence of diabetes mellitus (Rev Méd Chile 2005; 133:1311-6).

(Key Words: Diabetes mellitus; Hemochromatosis; Hemosiderosis; Hepatitis C, chronic)

Recibido el 6 de septiembre, 2004. Aceptado el 13 de junio, 2005.

Sección de Gastroenterología1, Departamentos de Medicina ${ }^{2}$ y de Anatomía Patológica ${ }^{3}$, Hospital Clínico Universidad de Chile. Departamento de Gastroenterología, Clínica Las Condes ${ }^{4}$. Santiago, Chile.

Correspondencia a: Dr. Javier Brahm B. Sección de Gastroenterología, Hospital Clínico Universidad de Chile. Santos Dumont 999, Independencia. Fono: 9788350. Fax: 9788349. E mail: jbrahm@redclinicauchile.cl 
E virus de la hepatitis C (VHC) infecta alrededor de 170 millones de personas en todo el mundo y representa una pandemia cinco veces mayor que la infección con el virus de la inmunodeficiencia humana (VIH). Más de $70 \%$ de los casos evolucionan a la cronicidad, con el desarrollo de cirrosis en $20 \%$, entre 10 y 20 años ${ }^{1,2}$. Además, el VHC es la principal causa de hepatitis crónica y de trasplante hepático en adultos en la actualidad $^{2}$.

Los factores asociados a la progresión del daño histológico del VHC no son claramente conocidos. Entre éstos se han señalado la edad, condición étnica, sexo masculino, antigüedad de la infección, respuesta inmune celular específica y genotipo del VHC, ingesta de alcohol, coinfección con VIH o virus hepatitis B (VHB), factores ambientales y geográficos, depósitos de hierro, diabetes mellitus (DM), sobrepeso (índice de masa corporal $>27 \mathrm{~kg} / \mathrm{m}^{2}$ ) y severidad de la inflamación y fibrosis en la biopsia hepática ${ }^{3}$.

Como estos factores no han sido estudiados en nuestro medio, el objetivo de este trabajo fue correlacionar la severidad del daño histológico en pacientes infectados por VHC, con la presencia de esteatosis y hemosiderosis en la biopsia hepática y su asociación con otros factores tales como edad, sobrepeso, diabetes y consumo de alcohol.

\section{PACIENTES Y MÉTODO}

Se realizó un estudio retrospectivo en 96 biopsias hepáticas de pacientes portadores de infección crónica por VHC, que se controlaban en los Servicios de Gastroenterología del Hospital Clínico de la Universidad de Chile y de la Clínica Las Condes, entre 1998 y 2002.

De estos registros se incluyeron finalmente 84 biopsias, 43 hombres y 41 mujeres, con promedio de 46 años (rango 20 a 72 años), los que tenían datos clínicos completos.

El análisis y revisión histológica de todos los casos fue realizada por uno de los coautores (GS), quien evaluó la actividad inflamatoria y fibrosis según los criterios de Knodell ${ }^{4}$ y los clasificó en tres grupos de hepatitis crónica: estadio I: hepatitis crónica con actividad leve y sin fibrosis; estadio II: hepatitis crónica con leve o moderada inflamación y fibrosis leve y estadio III: hepatitis crónica moderada con fibrosis severa o cirrosis. Además, se evaluó el grado de esteatosis y hemosiderosis en el tejido hepático.

La esteatosis se clasificó en grados según el porcentaje de hepatocitos comprometidos:

Grado 0: esteatosis menor a 5\%; grado 1: entre $5 \%$ y $30 \%$; grado 2 : mayor de $30 \%$ y menor de $60 \%$ y grado 3: mayor de 60\%. La hemosiderosis se clasificó en grados según la identificación de gránulos teñidos con azul de Prusia con el tamaño del campo visual: grado 0, gránulos ausentes o difícilmente identificables con aumento 400x; grado 1, gránulos difíciles de identificar con aumento 250x y fácilmente identificables con 400x; grado 2, gránulos observables con 100x; grado 3, gránulos observables con $25 \mathrm{x}$ y grado 4 , gránulos visibles con 10x o directamente ${ }^{5}$. También se analizó la asociación con la edad, presencia de sobrepeso (IMC $>27 \mathrm{~kg} / \mathrm{m}^{2}$ ), de obesidad, de diabetes mellitus (definida por glicemia en ayunas $>110 \mathrm{mg} / \mathrm{dl}$ ) y con la ingesta de alcohol (mayor o menor a 40 g/día). Para el análisis de las múltiples variables entre la esteatosis y hemosiderosis en los diferentes estadios histológicos de hepatitis crónica y su asociación con los factores clínicos estudiados, se utilizó el test exacto de Fisher.

\section{RESUlTADOs}

De los 84 pacientes con hepatitis crónica por VHC estudiados, el análisis histológico demostró que $41 \%$ se encontraba en estadio I, 32\% en estadio II y $27 \%$ en estadio III y su distribución según edad y sexo se observan en la Tabla 1. Los pacientes en estadio I tenían una edad $\overline{\mathrm{x}}$ significativamente menor que los estadios II y III, 40,7 años y 52,2 años respectivamente $(\mathrm{p}<0,001)$ y no tuvieron diferencias significativas en relación al sexo en los tres grupos estudiados ( $p>0,600)$ (Tabla 1).

No se demostró asociación entre los estadios histológicos y el grado de esteatosis $(p=0,206)$ y de hemosiderosis $(p=0,423)$ en la biopsia hepática, además en ningún caso se encontró hemosiderosis grado 4. Tampoco hubo asociación entre la severidad de la alteración de la biopsia y el IMC ( $\mathrm{p}$ $>0,217)$ y la ingesta del alcohol $(p>0,375)$. Sin embargo, la presencia de diabetes mellitus como variable independiente, se asoció a mayor severidad del daño histológico: 3\% de diabéticos en 
estadio I, $15 \%$ en estadio II y $30 \%$ en estadio III ( $p$ $<0,05)$. No se demostró una asociación significativa entre diabetes mellitus, esteatosis y hemosiderosis $(\mathrm{p}=0,854)$ (Tabla 2$)$.

\section{DisCUSIÓN}

En nuestro estudio encontramos, tal como era esperable, una asociación entre la edad y el estadio histológico, más avanzado en casos de hepatitis crónica por VHC, mostrando que a mayor edad es mayor el número de pacientes que presenta cirrosis. La diferencia de edad entre el estadio I con los II y III fue de aproximadamente 12 años. Como se describe en la literatura, el tiempo estimado para que la infección crónica por VHC evoluciona a hepatitis crónica y cirrosis es variable, con un promedio de 10 a 20 años, respectivamente ${ }^{1-3}$. Además, no se demostró diferencias significativas en relación al sexo en los diferentes grupos analizados. Por otra parte, encontramos que 3\% de los pacientes en estadio I presentaron diabetes, mientras que en el estadio II y III el porcentaje fue de 15 y $30 \%$, respectivamente ( $p=0,0045)$.

Se sabe actualmente que los pacientes con DM tipo 2 tienen una mayor prevalencia de cirrosis y, por otra parte, una proporción de pacientes con enfermedad hepática crónica desarmollan $\mathrm{DM}^{6-8}$. Además, hay datos emergentes que sugieren que la infección por VHC puede contribuir al desarrollo de DM. La intolerancia a la glucosa es más frecuente en pacientes con infección por VHC, comparada con casos controles con enfermedad hepática de otras causas $^{8}$. La frecuencia de infección por VHC en poblaciones europeas con DM tipo 2 se ha comunicado mayor que en la población general ${ }^{9-11}$.

Existen algunas comunicaciones que indican que la DM y estados de hiperinsulinemia pueden causar una progresión significativa del curso clíni-

Tabla 1. H epatitis crónica por VH C : C aracterísticas demográficas según severidad del daño histológico

\begin{tabular}{|lccc|}
\hline Estadio histológico & (n) & $\begin{array}{c}\text { Promedio edad } \\
\text { (rango) }\end{array}$ & Hombres/Mujeres \\
\hline I: Inflamación leve y sin fibrosis & $(34)$ & $41(20-61)^{1}$ & $15 / 19^{2}$ \\
II: Inflamación leve a moderada y fibrosis leve & $(27)$ & $51(33-69)^{1}$ & $15 / 12^{2}$ \\
III: Inflamación severa con fibrosis severa o cirrosis & $(23)$ & $55(37-72)^{1}$ & $13 / 10^{2}$ \\
Total & (84) & $49(20-72)$ & $43 / 41$ \\
\hline
\end{tabular}

${ }^{1}$ Estadio I vs II-III $=\mathrm{p}<0,001$

${ }^{2} \mathrm{NS}$

Tabla 2. G rupos histológicos de hepatitis crónica por VH C y su correlación con el grado de esteatosis y hemosiderosis en la biopsia hepática y con la presencia de sobrepeso/obesidad (IM C >27), ingesta exagerada de alcohol y diabetes mellitus

\begin{tabular}{|c|c|c|c|c|c|c|c|c|c|}
\hline \multirow[t]{2}{*}{$\begin{array}{l}\text { Estadios } \\
\mathrm{N}=84(\%)\end{array}$} & \multicolumn{3}{|c|}{$\begin{array}{c}\text { Esteatosis }^{1} \\
\text { hepática (\%) }\end{array}$} & \multicolumn{3}{|c|}{$\begin{array}{c}\text { Hemosiderosis }^{2} \\
\text { hepática (\%) }\end{array}$} & \multirow[t]{2}{*}{$\begin{array}{c}\mathrm{IMC}^{3}>27 \\
(\%)\end{array}$} & \multirow{2}{*}{$\begin{array}{c}\text { Alcohol }^{4} \\
>40 \text { grs/día } \\
\text { (\%) }\end{array}$} & \multirow{2}{*}{$\begin{array}{c}\text { Diabetes }^{5} \\
\text { Mellitus } \\
(\%)\end{array}$} \\
\hline & 0 & 1 & $2-3$ & 0 & 1 & $2-3$ & & & \\
\hline $\mathrm{I}=34(41)$ & 44 & 53 & 3 & 82 & 15 & 3 & 56 & 38 & 3 \\
\hline II = 27 (32) & 30 & 59 & 11 & 74 & 22 & 4 & 68 & 52 & 15 \\
\hline III = 23 (27) & 22 & 61 & 17 & 78 & 4 & 18 & 76 & 57 & 30 \\
\hline
\end{tabular}

$1,2,3,4 \mathrm{NS}$

${ }^{5}$ Estadio I-II vs III P $<0,05$ 
co de la hepatitis crónica por VHC, contribuyendo a favorecer la evolución a cirrosis ${ }^{12}$.

En el presente trabajo, la asociación entre DM y severidad de la esteatosis y hemosiderosis en la biopsia hepática no demostró ser significativa.

Del mismo modo, no se encontró una relación entre el grado de esteatosis y la severidad de la lesión histológica a la biopsia. No existen trabajos que evalúen en el tiempo esta variable en forma independiente. Lo que sí está descrito es que la esteatosis hepática parece jugar un rol central en la patogénesis de la enfermedad hepática en hepatitis crónica por VHC y puede ser causada tanto por factores virales como metabólicos ${ }^{12-14}$ (Figura 1).

Recientes estudios señalan que la esteatosis es más prevalente en la infección por el genotipo 3 comparado con genotipo 1 y $2^{15-17}$. Moriya et al, encontraron que la proteína del core y NS5A del VHC induce esteatosis en ratones transgénicos, sugiriendo que ésta podría tener un efecto directo en la infección por el VHC. Estas proteínas interfieren con la respuesta intracelular, el transporte de triglicéridos y el montaje y secreción de lipoproteínas ${ }^{18}$. Los procesos que llevan a la

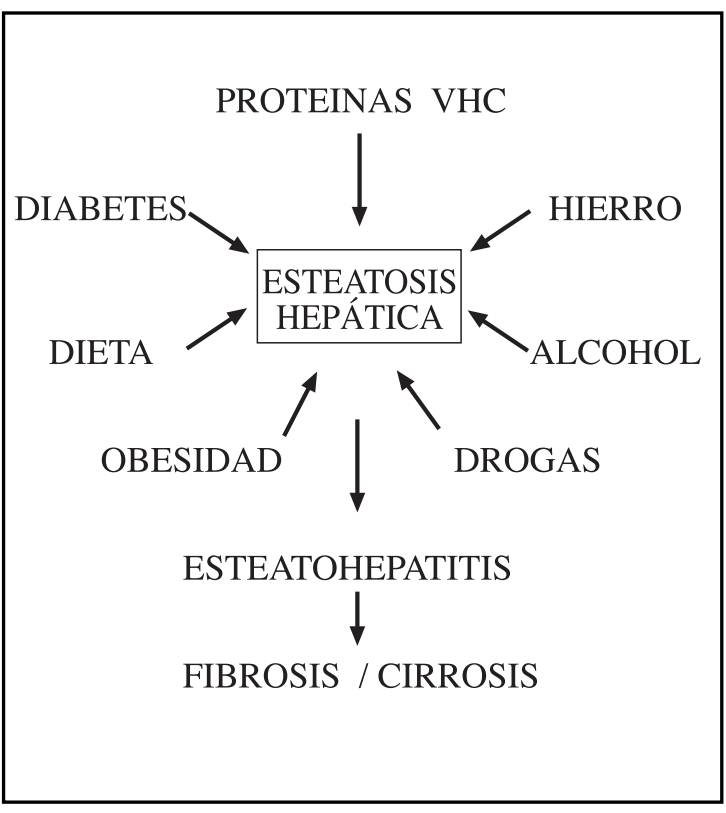

FIGURA 1. Mecanismos multifactoriales que contribuyen a la esteatohepatitis, en casos con hepatitis crónica por VHC. acumulación lipídica en el hepatocito de pacientes infectados por VHC parecen ser multifactorial. En un subgrupo de pacientes con esteatosis, se ha asociado a otros factores de hígado graso no alcohólico (HGNA) como la obesidad, DM e hiperinsulinemia ${ }^{19-21 .}$

La asociación entre obesidad y severidad del daño hepático en la biopsia en este estudio no demostró ser significativa. Aun así, creemos que se debería recomendar a los pacientes con enfermedad crónica por VHC un cambio en el estilo de vida, el cual incluye una reducción programada y sostenida del peso. En este sentido, existen trabajos con pocos pacientes y con períodos cortos de seguimiento, que demuestran una mejoría de las aminotransferasas y de la biopsia hepática en pacientes con infección crónica por VHC, que lograron una reducción significativa de peso ${ }^{22}$.

En el presente estudio no se encontró una asociación directa entre el consumo de alcohol y la severidad histológica, así como tampoco se demostró asociación entre la severidad de la esteatosis y el abuso del alcohol. Esto se opone a lo publicado, ya que la literatura muestra que el consumo de alcohol en forma habitual ( $>40$ a 50 gramos/día) favorece la progresión de fibrosis en pacientes con infección crónica por $\mathrm{VHC}^{23}$. El mecanismo fibrogénico del alcohol en casos de hepatitis crónica $\mathrm{C}$ es probablemente multifactorial. Así, existe una mayor viremia y carga viral asociada con el consumo de alcohol y fluctuación de la carga viral con períodos de ingesta de alcohol y de abstinencia 24,25 .

Por otra parte, los bebedores exagerados tienen un aumento del hierro hepático en casos de infección crónica $\mathrm{C}$, el cual se asocia con aumento de fibrosis ${ }^{26}$. Por lo tanto y a pesar de nuestros resultados, se debería recomendar a los pacientes con enfermedad por VHC la abstinencia de alcohol.

En nuestro trabajo no se encontró diferencia significativa entre el grado de hemosiderosis y la mayor severidad histológica de los distintos grupos analizados. Además, tampoco hubo relación entre el consumo de alcohol y la hemosiderosis en la biopsia.

Existe interés en el rol del hierro en la patogénesis de la infección crónica por VHC y su depleción como un tratamiento adjunto para evitar la progresión del daño hepático. 
Algunos estudios han demostrado por otra parte, que el exceso del hierro hepático disminuye la probabilidad de respuesta al tratamiento con interferon en pacientes con infección crónica del $\mathrm{VHC}^{27}$.

Por otra parte, ante la presencia de un grado mayor de hierro en el tejido hepático, se debe descartar la presencia de una mutación del gen de la hemocromatosis ${ }^{28-30}$. Si éste se confirma, el tratamiento indicado será la flebotomía previo al tratamiento, hasta que el paciente presente déficit relativo de hierro.

\section{REFERENCIAS}

1. Tong MJ, FarRa NS, Reikes AR, Co RL. Clinical outcomes after transfusion- associated hepatitis C. N Engl J Med 1995; 332: 1463-6.

2. TaKAHASHi M, Yamada G, Miyamoto R. Natural course of chronic hepatitis C. Am J Gastroenterol 1993; 88: 240-3.

3. Yano M, Kumada $H$, Kage $M$. The long-term pathological evolution of chronic hepatitis C. Hepatology 1996; 23: 1334-40.

4. Knodell RG, Ishak KG, Biack WC, Chen TS, Craig R, KaPLowitz N ET AL. Formulation and application of a numerical scoring system for assessing histological activity in asymptomatic chronic active hepatitis. Hepatology 1981; 1: 431-5.

5. Macsween RN, Burt AD, Portmann B, Ishak KG, Scheuer PJ, Anthony P. Iron stores diseases. eds. Pathology of the liver, $4^{\text {th }}$ edn. Edinburgh: Churchill Livingstone, 2002.

6. Petrides AS. Liver disease and diabetes mellitus. Diabetes Rev 1994; 2: 2-18.

7. Muting D, Wohlgemuth D, Dorsett R. Liver cirrhosis and diabetes mellitus. Geriatrics 1969; 24: 91-9.

8. Niedereau C, Fischer R, Sonnenberg A, Stremmel W, TRAmpisch NH, Strohmeyer G. Survival and causes of death in cirrhotic and non-cirrhotic patients with primary hemochromatosis. N Engl J Med 1985; 313: 1256-62.

9. Alison M, Wreghitt T, Palmer CR, Alexander GJM. Evidence for a link between hepatitis $C$ virus infection and diabetes mellitus in a cirrhotic population. J Hepatol 1994; 21: 1135-9.

10. OzyILKAN E, ARSLAN M. Increased prevalence of diabetes mellitus in patients with chronic hepati-
En suma, nuestra experiencia parecería confirmar que existe una correlación proporcional entre severidad histológica, mayor edad y mayor tiempo de infección en pacientes con hepatitis crónica por VHC y que, además, se asocia con diabetes mellitus. No encontramos una asociación entre la magnitud del daño hepático con el grado de hemosiderosis, el IMC y la ingesta de alcohol. Sin embargo, creemos que en estos pacientes deberían evitarse o controlar estos cofactores, los que en asociación con el VHC, pueden contribuir a aumentar el daño del hepatocito.

tis C virus infection. Am J Gastroenterol 1996; 91: 1480-1.

11. Mason AL, Lau JY, Hoang N, Qian K, Aiexander GJ, Xu L ET AL Association of diabetes mellitus and chronic hepatitis C vinus infection. Hepatology 1999; 29: 328-33.

12. Alberti A, Benvegnu L. Management of hepatitis C. J Hepatol 2003; 38: 104-18.

13. Scheuer PJ, Ashrafzadeh P, Sherlock S, Brown D, Dusheiko GM. The pathology of hepatitis C. Hepatology 1992; 15: 567-71.

14. Bach N, Thung SN, SchafFner F. The histological features of chronic hepatitis $\mathrm{C}$ and autoimmune chronic hepatitis: a comparative analysis. Hepatology 1992; 15: 572-7.

15. Minm S, Fayyazi A, HaRtmann H, Ramadori G. Analysis of histopathological manifestations of chronic hepatitis $\mathrm{C}$ virus infection with respect to virus genotype. Hepatology 1997; 25: 735-9.

16. Adinolfi LE, UtiL R, Adreana A, Tripodi MF, Rosario P, Mormone G ET AL. Relationship between genotypes of hepatitis $C$ virus and histopathological manifestations in chronic hepatitis $\mathrm{C}$ patients. Eur J Gastroenterol Hepatol 2000; 12: 299-304.

17. Rubbia-Brandt L, Quadri R, Abid K, Giostra E, Male PJ, Mentha G et al. Hepatocyte steatosis is a cytopathic effect of hepatitis $C$ virus genotype 3 . J Hepatol 2000; 33: 106-15.

18. Moriya $\mathrm{K}$, Yotsuyanagi $H$, Shintani $Y$, Fujie $H$, ISHiBASHI $\mathrm{K}$, MatsuURA $\mathrm{Y}$ ET AL. Hepatitis $\mathrm{C}$ virus core protein induces hepatic steatosis in transgenic mice. J Gen Virol 1997; 78: 1527-31.

19. Hourigan LF, MacDonaid GA, Purdie D, Whiteall VH, Shorthouse C, Clouston A et al. Fibrosis in chronic hepatitis $\mathrm{C}$ correlates significantly with body mass index and steatosis. Hepatology 1999; 29: 1215-9. 
20. Ong JP, Younossi ZM, Speer C, Olano A, Gramlich T, Boparai N. Chronic hepatitis $C$ and superimposed non-alcoholic fatty liver disease. Liver 2001; 21: 266-71.

21. Muzzi A, Leandro G, Rubbia-Brandt L, James R, KeISER O, MALINVERNI R ET AL. Insulin resistance is associated with liver fibrosis in non-diabetic chronic hepatitis C patients. J Hepatol 2005; 42: 41-6.

22. Hickman IJ, Clouston AD, MacDonald GA, Purdie DM, PRIS JB, Ash S ET aL. The effect of weight reduction on liver histology and biochemistry in patiens with chronic hepatitis. Gut 2002; 51: 8994.

23. Poynard T, Ratziu V, Charlotte F. Rates and risk factors of liver fibrosis progression in patients with chronic hepatitis C. J Hepatol 2001; 34: 7309 .

24. Everhart J, Herion D. Hepatitis C virus infection and alcohol. Liang TJ, Hoofnagle JH, eds. Hepatitis C. San Diego: Academic Press, 2000; 363-88.
25. Pessione F, Degos F, Marcein P. Effect of alcohol consumption on serum hepatitis C. Hepatology 1998; 27: 1717-22.

26. Britton RS, Ramm GA, Olyny J. Pathophysiology of iron toxicity. Adv Exp Med Biol 1994; 356: 239-53.

27. Poniachik J, Hurtado C, Brahm J. Niveles elevados de ferritina y saturación de transferrina en casos de necrosis hepatocelular severa. Gastroenterol Latinoam 1998; 9: 259 (Abst).

28. Thorburn D, Curry G, Spooner R, Spence E, Oien K, HALs D ET AL The role of iron and haemochromatosis gene mutations in the progression of liver disease in chronic hepatitis C. Gut 2002; 50: 248-52.

29. Smith B, Grove J, Guzail M, Day CP, Daly A, Burt A ET AL. Heterozygosity for hereditary hemochromatosis is associated with more fibrosis in chronic hepatitis C. Hepatology 1998; 27: 1695-9.

30. Negro F, Samil K, Rubbia-Brandt L, Quadri R, Male JP, ZARSKI JP ET AL. Hemochromatosis gene mutation in chronic hepatitis $C$ patients and without liver siderosis. J Med Virol 2000; 60: 21-7. 